\title{
Assessing alternative management strategies for blacklip abalone
}

\author{
$\underline{\text { R.A. Bedford }}^{\text {a }}$, J.W. Hearne ${ }^{\text {a }}$, Y. Wang ${ }^{\text {a }}$ and H.K. Gorfine ${ }^{\text {b }}$ \\ ${ }^{a}$ School of Mathematical and Geospatial Sciences, RMIT University, 124 La Trobe Street, Melbourne, \\ Victoria 3001, Australia \\ ${ }^{b}$ Department of Primary Industries, DPI Queenscliff Centre, Queenscliff, Victoria 3225, Australia
}

\section{E-mail: rbdfrd83@gmail.com}

\begin{abstract}
Unsustainability of abalone harvesting has become a worldwide trend. Australian abalone fisheries have thus far defied this seemingly inevitable path towards collapse, remaining generally sustainable until recently. Today unfavourable market and export conditions, disease, climate change, ecological shifts, and reduced catch quotas challenge the capacity of Australian abalone fisheries to remain sustainable into the future. Profits are down and the resilience of these fisheries is under threat.

The two major stakeholders of the Australian abalone fishing industry are the commercial license owners and the government. The licence owner's goal is to maximise their annual catch whilst the main responsibility of government is to ensure the continued health of the fishery. In the early decades of the fishery both goals were relatively easily satisfied, however recent times have seen increased conflict between the two parties. Government is obliged to strongly consider the livelihoods of the licence owners whenever it applies annual changes to commercial harvesting strategies. However, it is faced with increasingly difficult decisions as abalone populations spiral into decline.
\end{abstract}

Management of abalone fisheries has proved to be an onerous task over many decades. Abalone live near the shoreline and can be found down to 30 metres depth. Their populations from one reef to the next are as unpredictable as the rocky reefs they inhabit. Whilst many abalone are visible on the outer surface of a complex boulder system, it is difficult to accurately predict how many are hidden in inaccessible cracks and crevices.

Initially, fishery assessment modelling of abalone populations took place at a management zone level covering hundreds of kilometres of coastline. Due to recent factors such as population collapse from overfishing and disease, causing reduction of harvesting quotas, abalone biomass modelling is now performed at a finer regional level scale (a few kilometres of coastline). Single reefs, which provide a few tonnes of commercial catch annually, are now monitored in isolation. Due to the expensive nature of fishery independent sampling, most reefs rely primarily on the previous annual catch as the feedback source. Regular workshop meetings are a secondary feedback source, where diver observations at each reef are recorded. CPUE (catch per unit of effort) has been dismissed as having low efficacy in abalone fisheries due to divers' abilities to target multiple abalone aggregations efficiently, whilst the fishery is reasonably abundant. However, the importance of measuring effort from a modelling perspective is critical, when finding the tipping point, at which time a reef becomes unfishable due to scarcity of legal sized abalone.

This study explores the contrast between current management strategies and alternative management strategies incorporating indexes such as average length of commercial catch (ALCC) as a feedback. A central question in this process is; which management strategy is most likely to recover a vulnerable population, ensuring a robust biomass producing stable harvest rates? An existing Abalone model is used to test several harvest management strategies on a regional population of blacklip abalone, Halitois rubra. A high-mortality event is contrived early in the test phase, to simulate a disease sweeping through the population.

To assess long-term sustainability of the population, mean values of cumulative yield, total biomass and legal biomass are estimated at the conclusion of a simulation period. An average length of commercial catch [ALCC] strategy provided the longest-term sustainability, in the form of protection to legal biomass, for a population subjected to significant stock loss. However, the slow nature of harvest recovery under the ALCC strategy meant the existing harvesting strategy is the most economically viable strategy for our population recovery. Despite this finding, the abalone industry would benefit greatly by investing effort, measuring shell lengths among commercial catches at fine scales. These data would provide information resulting in greater protection to individual populations via the setting of appropriate legal minimum lengths.

Keywords: Legal minimum length, simulation, management strategy evaluation, blacklip abalone, sustainability 


\section{INTRODUCTION}

Historically, wild abalone have always attracted a high unit price with relatively low overhead attached to their capture. This places management of wild abalone fisheries are under ever-increasing pressure to provide a platform for sustainability of abundance well into the future. The application of adaptive management methods to Australian abalone fisheries have occurred over the past decade to ensure the continuing viability of our abalone industry. In Victoria, a state in the south-east corner of Australia, Prince et al. [2008] applied a hierarchical decision-tree during fishery assessment workshops involving key stakeholders. The recent history of decision-making and progress in engaging abalone divers in fishery assessments, combined with the introduction of finer-scale adaptive management, is well documented by Gilmour [2011].

Governance of the abalone fishery is heavily burdened in the adaptive management process on two counts. Firstly, the most recent licence owners have paid a substantial premium for their commercial abalone licence and secondly, industry has directly and indirectly contributed vast sums of money towards abalone management research over many years. Therefore, when industry negotiates with Government, they feel completely justified with their claims and expectations from management. Naturally, government must be seen to have a sympathetic ear to industry, whilst making tough business decisions to ensure the future sustainability of wild abalone fishing. Licence owners are also compromised in the adaptive management process, as many of them are passionate about ensuring the continued health of the abalone fishery. Not only will a healthy fishery enhance their commercial gains, they are also keen to hand over a thriving fishery to future generations. However, others facing financial difficulty tend to push the envelope of sustainability in an attempt to survive financially (Gilmour et al. [2011]).

Several years ago, a novel outbreak of the disease, abalone viral ganglioneuritis (AVG), afflicted abalone in western Victoria (Corbeil et al. [2010], Gilmour et al. [2011]), decimating abalone stocks throughout most of this region of the state. Currently there are some early signs of recovery with numbers of juveniles observed on reefs during fishery independent surveys conducted in 2011 that were not apparent during the preceding 3-4 years. However, it will be several more years before these juveniles grow sufficiently large to recruit to the fishery.

The purpose of this study was to explore alternative management harvesting strategy options within the framework of an abalone fishery. In particular, the focus was to determine which management strategy best suits achievement of the recovery of an abalone fishery, following an event involving dramatic losses of stock. Consistency and magnitude of recovery harvest and to a lesser extent, estimated biomass levels, are critical measurements in this study. Rather than estimate key population parameters, the same set of parameters are applied to each strategy, in order to focus solely on strategy effects. The existing strategy, as well as each alternative strategy, takes into account the same rules belonging to the existing adaptive management process.

Mayfield [2010] provides the foundation for the commercial catch threshold strategy tested in this study. Sampling the length structure of commercial catch aboard divers' boats or upon returning to port vastly reduces the cost and uncertainty associated with fishery independent sampling on the seabed. Increasing legal minimum length of harvest, once legal biomass becomes critically low is another management strategy simulated in the modelling, with the strategy of closing the fishery for four months prior to spawning season being used as another alternative to the existing strategy. The rationale behind this final alternative is to boost numbers in the mature population prior to the commencement of spawning.

\section{MODEL FORMULATION.}

\subsection{Length based population model.}

The simulation modelling of alternative management strategies performed in this study uses abalone population dynamics developed in the length-based stock assessment model developed by Breen [2003]. The expected fully-grown length of a blacklip abalone in this study is set to $154 \mathrm{~mm}$, with a $10 \%$ coefficient of variance.

\subsection{Blacklip abalone characteristics.}

Recruitment into our length-based abalone model occurs at two years of age, since knowledge of abalone biomass below this age is not well understood, particularly in species attracted to cryptic habitat. A stochastic version of the von Bertalanffy (VBL) growth equation is used to handle the two-dimensional variation in individual abalone growth (mean $154 \mathrm{~mm}$ ) and growth rate (mean 0.18). Since blacklip abalone have a strong preference for inhabiting cryptic spaces, especially during the juvenile period of their life cycle, only abalone 
more than 1-2 centimetres above the legal minimum length (LML) are modelled as fully emergent and mature. Fishing abalone less than 1-2 centimetres above the LML is compromised by selectivity issues, as illustrated by the population distribution (year 2030) in Figure 1.

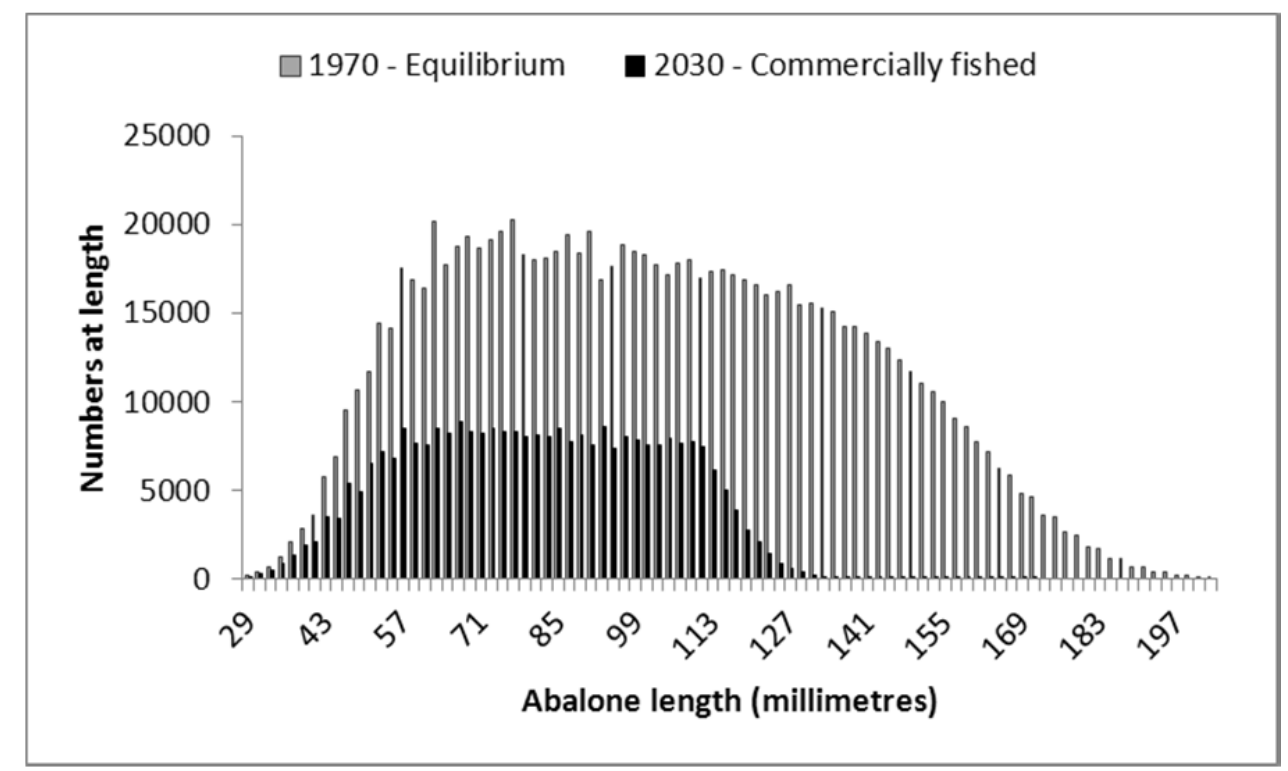

Figure 1. Equilibrium population (1970), prior to commercial fishing. The smaller shaded population (2030) demonstrates the effect of sixty years of commercial harvesting with the LML set to $110 \mathrm{~mm}$.

\section{MANAGEMENT STRATEGIES.}

\subsection{Average length of commercial catch [ALCC].}

With the advent of equipment used for electronically measuring and logging abalone shell size, it is now feasible to use the average shell length in the commercial catch as a performance measure (Mayfield [2010]). The ALCC strategy relies on three parameters to drive it. $L T$ and $U T$ are the lower and upper threshold lengths that the average length of catch is measured against and $\mathrm{r}$, where $0<r<1$, is the ratio of change in annual harvest to be applied whenever the average size falls outside the threshold values.

This method controls the composition of the mature abalone population by ensuring the average length lies in a chosen bandwidth. In this study $L T=110 \%$ of $L M L, U T=113 \%$ of LML and $r=0.9$. In essence the average length of the legal population oscillates between or just outside the lower and upper threshold lengths. When the average legal population length falls below the lower threshold, the previous years harvest is reduced by the change ratio $\mathrm{r}$. This protection given to the legal population gradually serves to raise the average harvest back between the threshhold values. Alternatively, when the average legal population length rises above the upper threshold limit, the previous years harvest is increased at the same change ratio $\mathrm{r}$. In this situation, the fishery is viable, thus reducing the risk attached to establishing harvest quotas.

\subsection{Existing methodology.}

Application of adaptive management processes in the Victorian abalone fishery is a relatively recent development. Currently, the associated rules are uncodified and not trivial to implement from a modelling perspective. In short, the existing harvesting and management practices at regional level are based around an optimal harvest target (OT), annual harvest upper limit (UL) and annual harvest lower limit (LL). Harvesting quota for the next fishing season is based on where last season's haul sits in relation to these three harvesting performance indicators.

\subsection{Legal Minimum Length.}

The third strategy under consideration within this model is the legal minimum length method. This method is also very similar to the existing method. The only difference is, that when legal abalone numbers fall below a tipping point (calclated by the model, see section 3.4) for the first time, the LML is raised to $116 \mathrm{~mm}$. Under this method, once the LML has been raised it remains at that level for the remainder of the simulation period. 


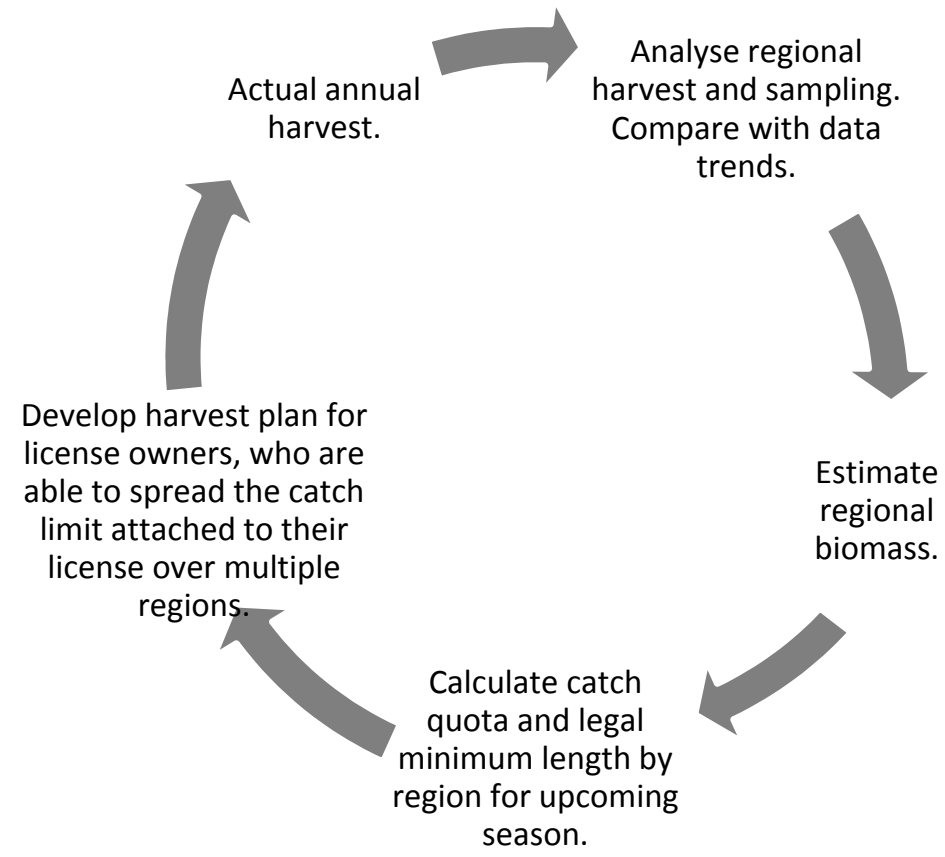

Figure 2. A typical fishery management cycle. Rather than estimate biomass, abalone management in Victoria uses commercial catch performance indicators OT, UL and LL (section 3.2) to facilitate the calculations of legal minimum length and quota for each region.

\subsection{Modelling problems.}

Ascertaining when the increasing effort required to obtain a catch causes the fishermen to go elsewhere to catch their quota is difficult to model. The tendency of the majority of abalone to form aggregations means that they are easy to locate until the overall population becomes heavily depleted. Furthermore, blacklip abalone are known to re-aggregate after fishing, with previously aggregated animals most likely to re-form an aggregation.

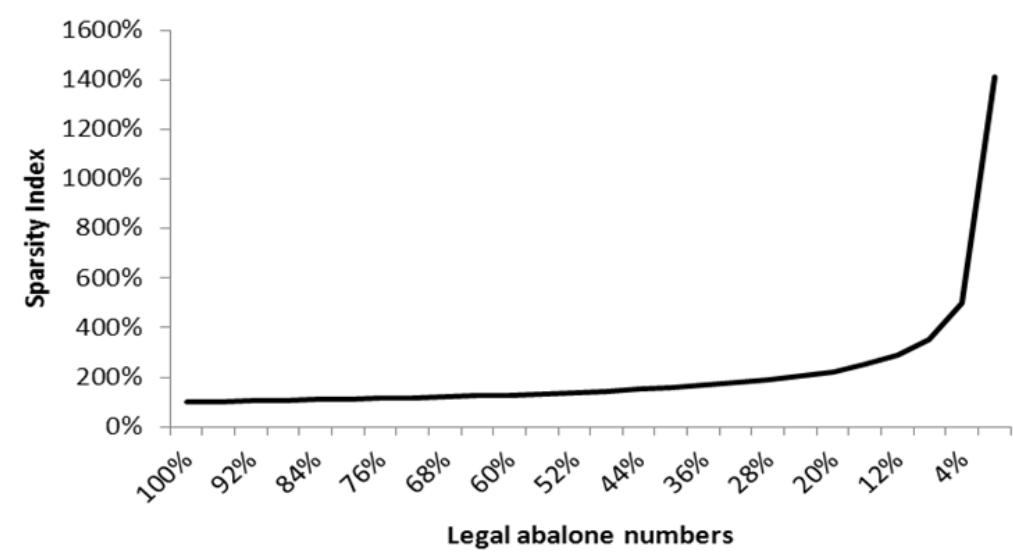

Figure 3. Increased fishing effort is a function of the declining numbers of legal abalone. The sparsity index, (proportion of equilibrium legal abalone numbers) ${ }^{-0.5}$, reflects the nature of abalone aggregation. When the legal population depletes to $4 \%$ of equilibrium legal population numbers a "tipping point" is reached.

Our model assumes the number of aggregations on a reef decreases at a similar rate to the average number of abalone within an aggregation. Therefore, aggregation density is measured as the square root of the proportion of equilibrium legal abalone numbers. Here, we model the sparsity of aggregations as an inversely proportional function of aggregation density (Figure 3).

After fishing ceases due to a scarcity of stock, modelling the point at which the fishery regains its perceived harvesting viability is also problematic. The point at which the legal abalone population exceeds $150 \%$ of the "tipping point" was arbitrarily chosen to be when the fishing would be re-commence. 


\section{RESULTS}

Simulation is applied to a reef in a stable state from year 2030. The objective here is to observe how the population under each harvesting strategy responds to an event involving dramatic loss of stock. In the 2nd year of simulation (2032) a shock impact is applied to the abalone population by increasing the instantaneous natural mortality rate from 0.2 to 0.6 . The harvest strategies are applied from this point until year 2060 .

The immediate impact of this high mortality event can be seen in Figure 4B, with stock biomass decreasing by approximately one third. Management reaction can be seen in Figure 4A, where the existing strategy produces three sharp harvesting recovery periods in the five years following the high mortality period (2032). Harvesting under the existing strategy reacts least to the population shock with short periods of moratoria, as the strategy is structured towards applying maximum pressure on the fishery. The ALCC strategy results in poor harvesting for more than a decade, followed by a strong recovery with the harvest oscillating towards an annual catch of 6 tonnes. The LML increase is invoked immediately under the LML strategy. This results in fishery closure due to insufficient legal biomass, followed by a consistent, albeit slow recovery. The biomass results (Figures $4 \mathrm{~B}$ and $4 \mathrm{C}$ ) represent the gains attributed to the conservative harvesting policies under the ALCC strategy and to a lesser extent, the LML strategy.

Cumulative harvesting under the ALCC strategy (Table 1) produced the most yield (142 tonnes), followed by the LML strategy (140 tonnes) with the existing strategy producing least harvest (132 tonnes). The stock biomass estimations at the conclusion of the simulation period in Table 1 show the existing strategy performs poorly with regard to the long term strength of the reef, in direct contrast to the ALCC method which provides most long-term stability. The ALCC strategy dramatically increases the original legal biomass of 3 tonnes over the thirty year period (Table 1), while the other fishing strategies leave the reef in a poor position in terms of short-term harvesting at the end of the simulation period.

A

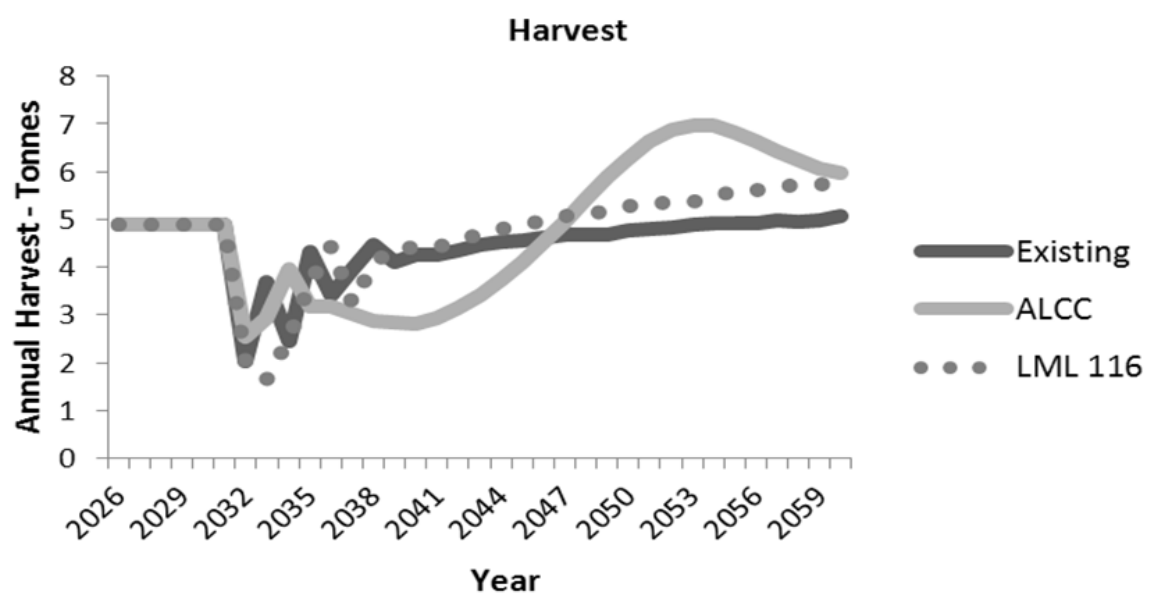

B

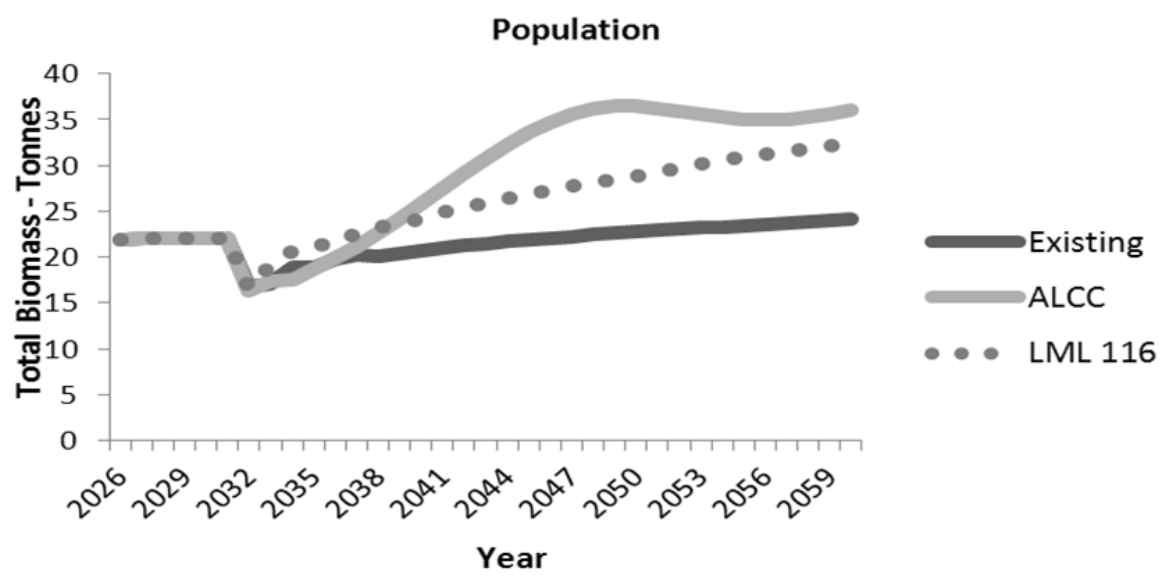


$\mathrm{C}$

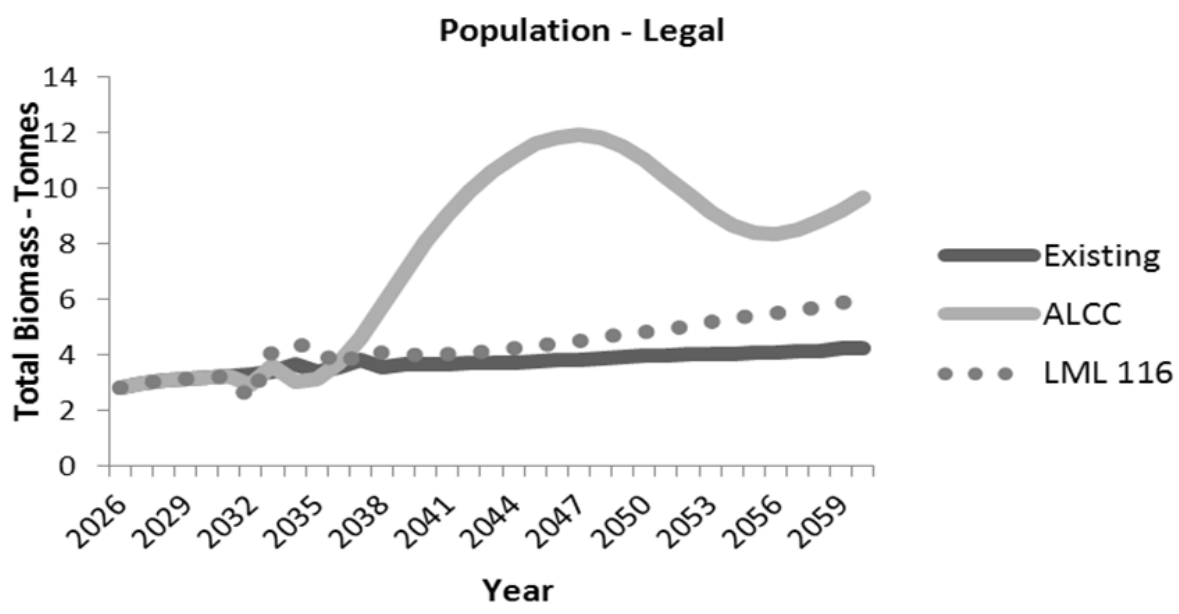

Figure 4. A) Mean annual harvest, B) mean population biomass and C) mean legal biomass simulation results under the three harvesting strategies (Section 3).

Stock is slow to recover $90 \%$ of the losses sustained in the second year of simulation, with an average recovery period of six years (Table 2). The harvest recovery under the ALCC strategy requires by far the longest recovery period (13.81 years). The legal population recovers quickest of the three measurements, which is understandable in the case of the LML strategy that results in no harvesting early in the recovery. In Table 2, the sustainability figures (in brackets) show the proportion of time the measurement stays above the $90 \%$ shock recovery point once reached. For example, the population stock recovery for the existing management strategy took 9.05 years and stayed above the recovery point $68.1 \%$ of the remaining 18.95 simulation years. The ALCC and LML strategies clearly have the most sustainable recoveries.

TABLE 1. Mean results for recovery simulation period 2031-2060 (represented in tonnes).

\begin{tabular}{llll} 
& Expected & Expected & Expected \\
& Cumulative & Biomass & Legal \\
& Harvest & 2050 & Biomass 2050 \\
Strategy & $(95 \%$ C.I $)$ & $(95 \%$ C.I $)$ & $(95 \%$ C.I $)$ \\
\hline Existing & $132.47(131.77,133.17)$ & $24.15(23.91,24.38)$ & $4.25(4.18,4.33)$ \\
ALCC & $142.36(141.42,143.31)$ & $36.05(35.64,36.46)$ & $9.65(9.43,9.87)$ \\
LML116mm & $140.00(139.33,140.68)$ & $32.50(32.14,32.86)$ & $6.02(5.86,6.17)$ \\
\hline
\end{tabular}

TABLE 2. Recovery [years (\% sustainability)] of $90 \%$ of losses in harvest, population biomass and legal biomass for current and alternative management strategies, during 2033-60 assuming $M=0.6$ during 2032.

\begin{tabular}{llll} 
Strategy & $\begin{array}{l}90 \% \text { harvest recovery } \\
\text { time in years }\end{array}$ & $\begin{array}{l}90 \% \text { total population } \\
\text { biomass recovery time } \\
\text { in years } \\
(\% \text { sustainability) }\end{array}$ & $\begin{array}{l}90 \% \text { legal biomass } \\
\text { recovery time in } \\
\text { years } \\
(\% \text { sustainability) }\end{array}$ \\
\hline Existing & $1.00(59.1 \%)$ & $9.05(68.1 \%)$ & $1.00(75.9 \%)$ \\
ALCC & $13.81(92.6 \%)$ & $5.81(99.7 \%)$ & $1.00(93.13 \%)$ \\
LML116mm & $2.82(72.5 \%)$ & $3.92(95.6 \%)$ & $1.00(94.4 \%)$
\end{tabular}

The immediate effect of the high-mortality event (2032) upon harvesting can be observed in Figure 5. In particular, the existing strategy experiences volatility in harvest due to multiple periods of fishing moratoria. 


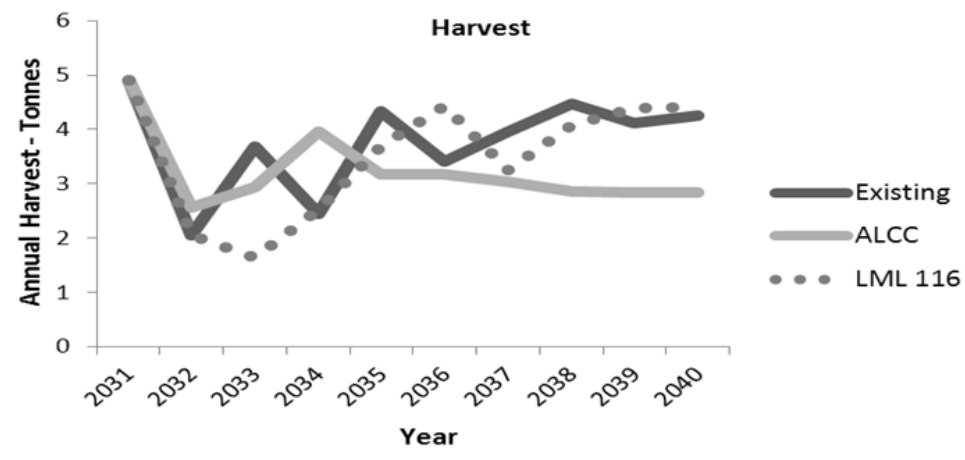

Figure 5. (Subset Figure 4A). Medium-term mean annual harvest after high-mortality event (2032).

\section{DISCUSSION.}

The primary goal of this study was to compare the existing Victorian abalone harvesting strategy with alternatives, to determine recovery performance in yield and stock biomass, following a high-mortality event. The existing strategy involves fishing down the population until a point where harvesting the amount of remaining legal animals requires too much effort for commercial viability. From this point onwards the reef remains unfished until the stocks regenerate to an "acceptable level" for resumption of harvesting.

In terms of volume and consistency of yield $(2031-2060)$, the existing strategy provided the best recovery in this study. Put simply, industry could ill afford the drawn-out harvest recoveries seen in Figure 4A, under the ALCC and LML strategies. However, it must be noted that none of the strategies provide a satisfactory consistency of yield in the first decade of recovery (Figure 5). The sharp rise and fall effect associated with the existing harvesting strategy recovery and the below-average harvesting yields of the ALCC and LML strategies would do irreparable damage to the livelihood of operators within the abalone industry.

The ALCC strategy provided the most significant boost to biomass levels because, firstly, since the average legal abalone size was already below LT prior to the high-mortality event, fishing pressure remained low for more than a decade, giving rise to long-term harvesting gains from these early savings. Also, the benefit of a two dimensional management strategy, using LML to decide legal abalone abundance in conjunction with average length of harvest specifications, provides extra protection to the legal stock. As a result, the ALCC strategy provided the most sustainable recovery, reaping the rewards of healthy harvesting into the future.

Increasing LML is a useful strategy which can generate stock growth and provide long-term harvesting benefits. Under the LML strategy, the cumulative harvest for the simulation period almost matched that of the ALCC strategy (Table 1). Despite a poor legal biomass (2060) figure under this strategy, it must be noted that the absence of animals in the $110-116 \mathrm{~mm}$ range has a downward effect on this figure. Increasing LML provided the quickest recovery periods (Table 2), because increasing the LML in effect, forces a de facto fishery closure during the period in which abalone grow to legal size.

For abalone populations recovering from disease, the existing management strategy provides a consistent harvesting recovery. However, for other struggling populations, management can take the inexpensive route and apply a trial and error approach with LML settings at a regional scale, in the hope that these populations will recover across the board. Alternatively, they can take the more sophisticated approach of data logging samples of the annual commercial catch, to assist in the setting of legal minimum lengths at regional scales.

\section{REFERENCES}

Breen, P. A., Kim, S. W., and Andrew, N. L. (2003), A length-based Bayesian stock assessment model for the New Zealand abalone Haliotis iris, Mar. Freshwater Res. 54, 619-634

Corbeil, S., Colling, A., Williams, L. M., Wong, F. Y. K., Savin, K., Warner, S., Murdoch, B., Cogan, N.O.I., Sawbridge, T. I. , Fegan, M., Mohammad, I., Sunarto, A., Handlinger, J. , Pyecroft, S., Douglas, M., Chang, P. H., St. J. Crane, M. (2010), Development and validation of a TaqMan ${ }^{\circledR}$ PCR assay for the Australian abalone herpes-like virus, Dis. Aquat. Org. 92, 1-10

Gilmour, P.W., Dwyer, P.D., and Day, R.W. (2011), Beyond individual quotas: The role of trust and cooperation in promoting stewardship of five Australian abalone fisheries, Mar. Pol. 35, 692-702.

Mayfield, S. (2010), Enhancing fishery assessments for an Australian abalone fishery using commercial weight-grade data, Fish. Res. 105, 28-37.

Prince, J. D., Peeters, H., Gorfine, H., and Day, R. W. (2008), The novel use of harvest policies and rapid visual assessment to manage spatially complex abalone resources (Genus Haliotis), Fish. Res. 94, 330-338. 